\title{
Communication of spin directions with product states and finite measurements
}

\author{
E. Bagan, M. Baig, and R. Muñoz-Tapia \\ Grup de Física Tè̀rica and IFAE, Facultat de Ciències, Edifici Cn, Universitat Autònoma de Barcelona, 08193 Bellaterra, Barcelona, \\ Spain
}

(Received 9 January 2001; published 5 July 2001)

\begin{abstract}
Total spin eigenstates can be used to intrinsically encode a direction, which can later be decoded by means of a quantum measurement. We study the optimal strategy that can be adopted if, as is likely in practical applications, only product states of $N$ spins are available. We obtain the asymptotic behavior of the average fidelity, which provides a proof that the optimal states must be entangled. We also give a prescription for constructing finite measurements for general encoding eigenstates.
\end{abstract}

DOI: 10.1103/PhysRevA.64.022305

PACS number(s): 03.67.Hk, 03.65.Ta

Quantum mechanics is rapidly broadening our knowledge of the ways information can be stored, transmitted, and retrieved. Here we address the concrete issue of communicating information of a direction using quantum states, which has attracted much attention [1-8]. Consider two parties, Alice and Bob, and imagine that Bob is lost in space and Alice wants to tell him the direction home. If communication by standard means is difficult, she can encode the direction in a quantum system and physically send it to Bob. Alice's quantum state must intrinsically point along the direction, given by the unit vector $\vec{n}$. If we assume that her system is made out of $N$ spins, then it must be an eigenstate of $\vec{n} \cdot \vec{S}$, where $\vec{S}$ is the total spin [9]. After he receives the state, Bob can perform a quantum measurement and retrieve Alice's direction with some accuracy. From each outcome (labeled with an index $r$ ) of the measurement, Bob will guess a direction, given by a unit vector $\vec{n}_{r}$. We use the fidelity (1 $+\vec{n} \cdot \vec{n}_{r}$ ) $/ 2$, as a figure of merit (we have also computed the information gain for a check of our conclusions). An average fidelity $F=1$ means a perfect determination of the direction. We can view $N$ as the size of the resources available to Alice. Obviously, the average fidelity should increase as the resources increase. However, for a given number of resources, the actual value of the average fidelity and the rate it approaches to one depend on the type of states being used. For instance, the maximal average fidelity (MAF) for states of $N$ parallel spins, $|\uparrow \uparrow \cdots \uparrow\rangle$, is $F=(N+1) /(N+2)$ [1], which is readily seen to approach unity linearly: $F \sim 1-1 / N$. If the resources consist of only two spins, choosing them to be antiparallel, $|\uparrow \downarrow\rangle,|\uparrow \downarrow\rangle$, leads to a value of [4] $F=(3$ $+\sqrt{3}) / 6$, which is larger than $F=3 / 4$ for two parallel spins. Thus, one can improve on the communication of a direction without increasing the resources. In Refs. [6,8] we generalized these results to arbitrary $N$ and computed the MAF optimizing both Alice's states and Bob's measurements. We proved that the MAF approaches unity as $F \sim 1-5.8 / N^{2}$, i.e., there is a quadratic improvement on the quality of the communication process over the parallel case. The optimal states that lead to such MAF are, in fact, a whole family of states, which for $N>2$ does not seem to include any state of the form $|\uparrow \downarrow \downarrow \cdots \uparrow\rangle$ (we will loosely refer to these states as product states). From the practical point of view, however, product states are of crucial importance, since they are likely to be the only ones that can be used in real devices (although they are expected not to be optimal). There are then two obvious questions one would like to answer. First, among these states, what are the best for encoding a direction? And, second, is there a quadratic improvement in the rate the MAF approaches to one? We will answer these questions in this paper. We show that the optimal product states are those with the smallest $|m|$, where $m$ is the eigenvalue of $\vec{n} \cdot \vec{S}$, and that the corresponding MAF for large $N$ is $F \sim 1-1 /(2 N)$. This result proves our implicit assumption that the truly optimal states are entangled for $N>2$. Although product states do not exhibit the quadratic behavior in $1 / N$ of the truly optimal ones, we see that they are still much better than the $N$ parallel spin states for communicating a spin direction.

To compute the MAF of an optimal measurement, it is useful to consider a positive operator valued measurement (POVM) with infinitely many outcomes or continuous POVM [10]. We show, however, that one can always construct optimal POVM's with a finite number of outcomes. This is an important point since these are the only measurements that can be physically implemented. For parallel encodings, there are explicit realizations of optimal finite POVM's for arbitrary $N$ [2], and minimal versions of these for $N \leqslant 7$ can be found in Ref. [3]. The outcomes of these POVM's are associated with unit vectors $\vec{n}_{r}$ (directions) that we can picture as the vertices of certain polyhedra inscribed in the unit sphere. In this paper we prove that the very same polyhedra define optimal measurements for very general encoding states and that the minimal polyhedra of Ref. [3] remain minimal for these general states.

Alice's states can be obtained by rotating a fixed eigenstate of $S_{z}=\vec{z} \cdot \vec{S}$ that we denote by $|A\rangle$. In terms of the individual spins it is just of the form $|\uparrow \downarrow \downarrow \uparrow \cdots\rangle$. It is convenient to write all quantum states in terms of the irreducible representations of $S U(2)$, thus

$$
|A\rangle=\sum_{j=m}^{N / 2}\left(\sum_{\alpha} A_{j}^{\alpha} \mid j, m ; \alpha\right),
$$

where the first two labels are the usual quantum numbers of the total spin $\vec{S}^{2}$ and its third component $S_{z}$, i.e., $\vec{S}^{2}|j, m ; \alpha\rangle=j(j+1)|j, m ; \alpha\rangle \quad$ and $S_{z}|j, m ; \alpha\rangle=m|j, m ; \alpha\rangle$. The third index $\alpha$ labels different occurrences of the same 
TABLE I. Maximal average fidelities $(F)$ and information gains $(I)$ for parallel $(P)$, antiparallel $(A)$ and optimal $(O)$ encodings.

\begin{tabular}{ccccccc}
\hline \hline$N$ & 2 & 3 & 4 & 5 & 6 & 7 \\
\hline$F_{P}$ & 0.75 & 0.8 & 0.8333 & 0.8571 & 0.875 & 0.8889 \\
$F_{A}$ & 0.7887 & 0.8444 & 0.8848 & 0.9069 & 0.9235 & 0.9342 \\
$F_{O}$ & 0.7887 & 0.8449 & 0.8873 & 0.9114 & 0.9306 & 0.9429 \\
& & & & & & \\
$I_{P}$ & 0.6232 & 0.9180 & 1.1678 & 1.3827 & 1.5708 & 1.7376 \\
$I_{A}$ & 0.8664 & 1.2816 & 1.7077 & 2.0079 & 2.2873 & 2.4897 \\
$I_{O}$ & 0.8664 & 1.2925 & 1.7589 & 2.1086 & 2.4685 & 2.7548 \\
\hline \hline
\end{tabular}

representation $\mathbf{j}$ in the Clebsch-Gordan decomposition of $(1 / 2)^{\otimes N}$. Also from Ref. [8], one can show that there exists an optimal continuous POVM, defined by a complete set of positive projectors of the form $O(\vec{n})=U(\vec{n})[|B\rangle\langle B$ $\left.+\left|B^{\prime}\right\rangle\left\langle B^{\prime}\right|+\cdots\right] U^{\dagger}(\vec{n})$, where $U(\vec{n})$ is the element of $S U(2)$ associated with the rotation $R: \vec{z} \mapsto \vec{n}$, and $|B\rangle,\left|B^{\prime}\right\rangle$, $\ldots$, are fixed states given by linear combinations entirely analogous to Eq. (1). The average fidelity is

$$
F=\int d n \frac{1+\vec{z} \cdot \vec{n}}{2}\langle A|O(\vec{n})| A\rangle .
$$

To compute Eq. (2) one can use just the effective state $|\widetilde{B}\rangle=\sum_{j=m}^{N / 2} \sqrt{2 j+1}|j, m\rangle$, instead of employing all of $|B\rangle$, $\left|B^{\prime}\right\rangle, \ldots$, i.e., $O(\vec{n}) \rightarrow U(\vec{n})|\widetilde{B}\rangle\langle\widetilde{B}| U^{\dagger}(\vec{n})$. Similarly, for given quantum numbers $j, m$, we define the effective components of $|A\rangle$ as $\widetilde{A}_{j} \equiv \sqrt{\Sigma_{\alpha}\left(A_{j}^{\alpha}\right)^{2}}$, which contains the information required to compute the MAF. For any $|A\rangle$ of the form $|\uparrow \downarrow \uparrow \cdots\rangle$ with $n_{\uparrow}$ spins up and $n_{\downarrow}$ spins down, the MAF in Eq. (2) can be computed using the effective state $|\widetilde{A}\rangle$ $=\sum_{j=m}^{N / 2} \widetilde{A}_{j}|j, m\rangle$, where $m=\left(n_{\uparrow}-n_{\downarrow}\right) / 2$ and the coefficients $\widetilde{A}_{j}$ are explicitly given by

$$
\widetilde{A}_{j}=\sqrt{\frac{1+2 j}{J+1+j}} \sqrt{\frac{(J-m) !(J+m) !}{(J-j) !(J+j) !}}, \quad J \equiv \frac{N}{2} .
$$

We obtain the following MAF:

$$
F=\frac{1}{2}+\frac{1}{2} \sum_{j=m}^{J} \mu_{j} \widetilde{A}_{j}^{2}+\sum_{j=m+1}^{J} \widetilde{A}_{j-1} \widetilde{A}_{j} \nu_{j},
$$

where [8] $\mu_{j}=m^{2} / j(j+1)$ and $\nu_{j}=j\left(j^{2}-m^{2}\right) / \sqrt{4 j^{2}-1}$.

We have written equal quantum numbers $m$ for $|A\rangle$ and $|B\rangle$. Note that if $m_{B}>m_{A}, O(\vec{n})$ would not be a complete set of projectors on the whole Hilbert space spanned by $U(\vec{n})|A\rangle$; conversely, if $m_{B}<m_{A}$, Alice's states do not use the full capabilities of Bob's measuring device and the strategy cannot be optimal.

The maximal fidelity in Eq. (4) is attained for the minimal value of $|m|$ (this is $m=0$ for $N$ even and $m=1 / 2$ for $N$ odd), i.e., for maximal antiparallel spins. In Table I we collect the values of the MAF for up to $N=7$ and we compare them with the MAF's of parallel $\left(F_{P}\right)[1]$ and optimal $\left(F_{O}\right)[8]$ encodings. Note that antiparallel product states lead to MAF's $\left(F_{A}\right)$ remarkably close to the optimal ones. Moreover, one can easily prove that antiparallel spins are better than parallel ones for encoding a direction. We now show this for an even number of spins, $N=2 n$, and $m=0$, in which case the MAF takes the simple form

$$
F_{A}=\frac{1}{2}+\sum_{j=1}^{n} \frac{n !^{2}}{(n-j) !(n+j) !} \frac{j}{\sqrt{(n+1)^{2}-j^{2}}} .
$$

Setting $j=0$ inside the square root, we obtain

$$
F_{A}>\frac{1}{2}+\sum_{j=1}^{n} \frac{n !^{2} j /(n+1)}{(n-j) !(n+j) !}=\frac{N+1}{N+2}=F_{P} .
$$

We would next like to study the large $N$ asymptotic behavior of $F_{A}$ to see whether it exhibits the quadratic behavior of the optimal states $1-F_{O} \sim 1 / N^{2}$. We just have to compute Eq. (5) for large $n$. Notice first that, using the Stirling approximation, we have the following limit:

$$
\frac{n !^{2}}{(n-j) !(n+j) !} \rightarrow e^{-j^{2} / n}\left(1+\frac{j^{2}}{2 n^{2}}-\frac{j^{4}}{6 n^{3}}+\cdots\right) .
$$

Therefore, only terms with $j \sim \sqrt{n}$ give a significant contribution to the sum in Eq. (5). Hence, it is legitimate to expand the square root in Eq. (5) in powers of $j$. The resulting expression can be evaluated by means of the Euler-Maclaurin formula [11]

$$
\begin{aligned}
\sum_{j=1}^{n} \frac{1}{n} f(j / n)= & \int_{0}^{1} d x f(x)+\frac{f(1)-f(0)}{2 n}+\frac{f^{\prime}(1)-f^{\prime}(0)}{12 n^{2}} \\
& -\cdots,
\end{aligned}
$$

where in our case $f(j / n)$ is the product of the right-hand side of Eq. (7) times the expansion of $j / \sqrt{(n+1)^{2}-j^{2}}$. Taking into account all the relevant terms, one obtains that up to order $1 / n$,

$$
F_{A}=1-\frac{1}{4 n}+\cdots=1-\frac{1}{2 N}+\cdots
$$

Therefore, antiparallel spin states lead to a MAF that approaches unity in $1 / N$, faster than it does for parallel spins, but only because of the smaller negative coefficient of the $1 / N$ term (1/2 compared to 1$)$. In this sense, both types of encodings are qualitatively similar. The quadratic behavior of truly optimal states (which are entangled) cannot be attained by any product state. It is lengthier, but straightforward, to compute the subleading term in Eq. (9). We obtain the following compact expression for the MAF:

$$
F_{A}=\frac{2 N+1}{2 N+2}+O\left(1 / N^{3}\right) \text {. }
$$

To check that our results are not an artifact of our particular figure of merit, we have also computed the average information gain [12], $I=\int d n\langle A|O(\vec{n})| A\rangle \log _{2}(\langle A|O(\vec{n})| A\rangle)$, for parallel, antiparallel, and optimal states. Our results are also 
collected in Table I. We see that both information gain and fidelity exhibit the same pattern. Namely, optimal (entangled) states lead to the largest $I$ and $F$, but antiparallel spins have values very close to the optimal ones and much larger than those of parallel spins.

Up to now we have dealt with continuous POVM's. They are useful mathematical tools that simplify the calculation of the MAF for any optimal measurement on an isotropic distribution of directions. The projectors $O(\vec{n})$ satisfy the closure relation $\int d n O(\vec{n})=\mathbb{I}$ because of the orthogonality of the nonequivalent irreducible $S U(2)$ representations, $\mathfrak{D}_{m m^{\prime}}^{(j)}$, under the isotropic integration over the unit sphere. However, only POVM's with a finite number of outcomes, can be realized in nature. Unfortunately, finite POVM's are rather elusive because there is no clear and unique definition of isotropy for a finite set of directions (unit vectors) $\vec{n}_{r}$. We provide here a functional definition, which will enable us to give a general algorithm for constructing optimal and finite POVM's. Moreover, it will become obvious that the problem of discretizing a POVM is of geometrical nature.

In the context of this paper, we say that a finite set of unit vectors $\vec{n}_{r}$ is isotropically distributed up to spin $J$ if there exist positive weights $\left\{c_{r}\right\}$ such that the following orthogonality relation holds for any $j, j^{\prime} \leqslant J$ :

$$
\sum_{r=1}^{N(J)} c_{r} \mathfrak{D}_{m k}^{(j)}\left(\vec{n}_{r}\right) \mathfrak{D}_{m^{\prime} k}^{\left(j^{\prime}\right) *}\left(\vec{n}_{r}\right)=\frac{C_{J}}{2 j+1} \delta_{m}^{m \prime \prime} \delta_{j}^{j \prime}
$$

where $C_{J}=\Sigma_{r=1}^{N(J)} c_{r}$ is the equivalent of the solid angle $4 \pi$ in the continuous orthogonality relation $\int d \Omega \mathfrak{D}_{m k}^{(j)}(\vec{n}) \mathfrak{D}_{m^{\prime} k}^{\left(j^{\prime}\right) *}(\vec{n})=4 \pi \delta_{m}^{m \prime} \delta_{j}^{j \prime} /(2 j+1)$, and $N(J)$ is the number of elements of $\left\{\vec{n}_{r}\right\}$. Here we use the shorthand notation $\quad \mathfrak{D}_{m k}^{(j)}(\vec{n})=\mathfrak{D}_{m k}^{(j)}(\phi, \theta, 0), \quad$ where $\quad \mathbf{n}$ $=(\sin \theta \cos \phi, \sin \theta \sin \phi, \cos \theta)$, and $\alpha, \quad \beta, \quad \gamma \quad$ in $\mathfrak{D}_{m k}^{(j)}(\alpha, \beta, \gamma)$, are the standard Euler angles. The main difference between the continuous orthogonality relation and Eq. (11) is that the latter can only hold for $j, j^{\prime}$ up to a maximal value $J$. The larger $J$ is, the larger the $N(J)$ that must be chosen.

We will now show that Eq. (11) is equivalent to

$$
\begin{aligned}
\sum_{r=1}^{N(J)} c_{r} Y_{L}^{M}\left(\theta_{r}, \phi_{r}\right)=0 ; \quad & =1,2, \ldots, 2 J \\
M & =0,1, \ldots, L,
\end{aligned}
$$

where $Y_{l}^{m}(\theta, \phi)$ are the standard spherical harmonics. Equation 12 is very appealing since one can establish a physical analogy. If we view $c_{r}$ as a (positive) charge at the position $\vec{n}_{r}$, Eqs. 12 tell us that Eq. (11) is equivalent to the requirement that electrostatic multipoles of order less or equal to $2 \mathrm{~J}$ vanish. Conditions (12) are exactly those given in Ref. [3] for minimal and optimal POVM's in the case of a signal state consisting of $N$ parallel spins. We see here that Eqs. (12) are actually of much greater generality. To simplify the notation, it is convenient to define the quantities

$$
z_{L}^{M}=\sum_{r}^{N(J)} c_{r} \mathfrak{D}_{M 0}^{(L) *}\left(\vec{n}_{r}\right)=\frac{\sqrt{4 \pi}}{\sqrt{2 L+1}} \sum_{r}^{N(J)} c_{r} Y_{L}^{-M}\left(\theta_{r}, \phi_{r}\right) .
$$

Now Eqs. (12) simply read $z_{L}^{M}=0$ for all $L$ and $M$ listed there. In the following, $j$ and $j^{\prime}$ are required to satisfy $j, j^{\prime}$ $\leqslant J$. The group theoretical results that will be used below are mainly borrowed from Ref. [13]. Note first that the product $\mathfrak{D}_{m k}^{(j)} \mathfrak{D}_{m^{\prime} k}^{\left(j^{\prime}\right) *}$ in Eq. (11) can be written as a sum of $\mathfrak{D}_{m-m^{\prime} 0}^{(l) *}$ $\propto z_{l}^{m-m^{\prime}}$. Explicitly, Eq. (11) is equivalent to the set of linear equations

$$
\begin{gathered}
\sum_{l}(2 l+1)\left(\begin{array}{ccc}
j & j^{\prime} & l \\
m & -m^{\prime} & m-m^{\prime}
\end{array}\right)\left(\begin{array}{ccc}
j & j^{\prime} & l \\
k & -k & 0
\end{array}\right) z_{l}^{m-m^{\prime}} \\
=(-1)^{m^{\prime}-k} \frac{C_{J}}{2 j+1} \delta_{m}^{n^{\prime}} \delta_{j}^{j \prime},
\end{gathered}
$$

where

$$
\left(\begin{array}{ccc}
j & j^{\prime} & j^{\prime \prime} \\
m & m^{\prime} & m^{\prime \prime}
\end{array}\right)
$$

are the $3-j$ symbols and the sum runs over all $l$ satisfying the triangular condition (in particular $l \leqslant 2 J$ ). By direct substitution, it is trivial to check that Eq. (12) is a solution of Eq. (13) for all relevant $j, j^{\prime}$ and $m, m^{\prime}$. Therefore, Eqs. (12) are sufficient conditions. To prove that Eqs. (12) are also necessary, we multiply Eq. (13) by

$$
\left(\begin{array}{ccc}
j & j^{\prime} & L \\
m & -m^{\prime} & M
\end{array}\right)
$$

and sum over $m$ and $m^{\prime}$. Next, we use the orthogonality condition [13]

$$
\sum_{m m^{\prime}}\left(\begin{array}{ccc}
j & j^{\prime} & l \\
m & m^{\prime} & k
\end{array}\right)\left(\begin{array}{ccc}
j & j^{\prime} & l^{\prime} \\
m & m^{\prime} & k^{\prime}
\end{array}\right)=\frac{\delta_{l}^{l^{\prime}} \delta_{k}^{k^{\prime}}}{2 l+1},
$$

where it is assumed that the triangular condition is satisfied, to obtain

$$
\left(\begin{array}{ccc}
j & j^{\prime} & L \\
k & -k & 0
\end{array}\right) z_{L}^{M}=(-1)^{-k}\left(\begin{array}{ccc}
j & j^{\prime} & 0 \\
0 & 0 & 0
\end{array}\right) C_{J} \delta_{j}^{j \prime} \delta_{L}^{0} \delta_{M}^{0} .
$$

Let us consider the possible cases in this equation separately. For $L \neq 0$, Eq. (15) is simply

$$
\left(\begin{array}{ccc}
j & j^{\prime} & L \\
k & -k & 0
\end{array}\right) z_{L}^{M}=0, \quad \forall j, j^{\prime} \leqslant J .
$$

The variables $z_{L}^{M}$ must be zero for $L=1,2, \ldots, 2 J$, since the $3-j$ symbols are nonvanishing. The other case, i.e., $L=0$, does not give further information about $z_{L}^{M}$, since the corresponding condition is trivially satisfied because of the properties of the 3-j symbols [13]. This completes the proof of the equivalence between Eqs. (11) and (12). 
From Eqs. (12), and working along the same lines as Derka et al. [2], one can produce an algorithm for finite POVM's. Suppose $J$ is integer (if it is not, consider the nearest integer $\hat{J}>J)$. We define $2 J+1$ angles $\phi_{s}=2 s \pi /(2 J$ $+1) ; s=0,1, \ldots, 2 J$. Then $\Sigma_{s=0}^{2 J} Y_{L}^{M}\left(\theta, \phi_{s}\right)=0(M>0)$ for any $\theta$, and we only need to solve

$$
\sum_{k} c_{k} P_{L}\left(\cos \theta_{k}\right)=0, \quad L=1,2, \ldots, 2 J,
$$

where $P_{L}$ is the Legendre polynomial of degree $L$. We choose $\theta_{k}$ to be the $2 J+2$ angles $\theta_{k}=k \pi /(2 J+1) ; k$ $=0,1, \ldots, 2 J+1$; and define $c_{0}=c_{2 J+1}=1$. Then, the system (17) of linear equations for $c_{1}, c_{2}, \ldots, c_{2 J}$ always has a positive solution. Actually, $c_{k}>1$ for $k=1,2, \ldots, 2 J$. To summarize, the unit vectors $\vec{n}_{r} \rightarrow \vec{n}_{k s}$ $=\left(\sin \theta_{k} \cos \phi_{s}, \sin \theta_{k} \sin \phi_{s}, \cos \theta_{k}\right)$, along with the corresponding weights $c_{r} \rightarrow c_{k s} \equiv c_{k}$ are isotropically distributed, i.e., Eq. (11) is satisfied.

The above algorithm enables us to discretize any optimal continuous POVM. Just take the very same state(s) $|B\rangle$ used to generate the projectors $O(\vec{n})$ and consider the new (finite) set $O\left(\vec{n}_{r}\right)=U\left(\vec{n}_{r}\right)|B\rangle\langle B| U^{\dagger}\left(\vec{n}_{r}\right)$. Modulo a trivial global normalization factor, $\left\{O\left(\vec{n}_{r}\right)\right\}$ defines a finite POVM. The finite measurement thus obtained leads to the same fidelity as the continuous one we started with. Moreover, since the conditions (12) are exactly those used in Ref. [3] to obtain minimal POVM's, it is clear that this construction also provides minimal POVM's for general $|B\rangle$ states. For instance, the minimal POVM for $N=2$ has four outcomes pointing to the vertices of a tetrahedron, while for $N=3$ there are six outcomes corresponding to the vertices of an octahedron.
Finally, we would like to note that, as far as the fidelity is concerned, Alice could also simulate a continuous isotropic distribution of directions by using a finite set $\left\{\vec{n}_{r}\right\}$ of isotropically distributed vectors (11) with a priori probability given by the weights $\left\{c_{r} / C_{J}\right\}$. The fidelity will not change provided $J \geqslant(2 j+1) / 2$, where $j$ is the total spin of the signal state ( $j=N / 2$ for a system of $N$ spins). For instance, if $N$ $=2$ and Alice uses unit vectors pointing to the vertices of an octahedron $(J=3 / 2)$ with equal probability $1 / 6$, the maximal fidelities will be precisely those shown in Table I for a truly (continuous) isotropic distribution, namely, $F_{P}=3 / 4$ and $F_{A}$ $=(3+\sqrt{3}) / 6$.

In summary, product states of antiparallel spins represent an excellent balance between feasibility of construction and capability to communicate spin directions. For small number of spins, their maximal fidelity is remarkably close to the maximal value that can be possibly achieved. For large $N$ these states lead to an average fidelity that approaches unity faster than states with parallel spins, although they do not exhibit the quadratic improvement of the optimal states. We have thus proven that the truly optimal encoding necessarily requires entanglement. We have also obtained a simple set of conditions for constructing finite measurements. These conditions work for any eigenstate of the total spin and, therefore, also hold for product states.

The authors thank R. Tarrach and A. Brey for their collaboration during the early stages of this work, and $\mathrm{M}$. Lavelle for a careful reading of the manuscript. Financial support from CICYT Contract No. AEN99-0766 and CIRIT Contract Nos. 1998SGR-00051 and 1999SGR-00097 is acknowledged.
[1] S. Massar and S. Popescu, Phys. Rev. Lett. 74, 1259 (1995).

[2] R. Derka, V. Buzek, and A. K. Ekert, Phys. Rev. Lett. 80, 1571 (1998)

[3] J. I. Latorre, P. Pascual, and R. Tarrach, Phys. Rev. Lett. 81, 1351 (1998).

[4] N. Gisin and S. Popescu, Phys. Rev. Lett. 83, 432 (1999).

[5] S. Massar, Phys. Rev. A 62, 040101(R) (2000).

[6] E. Bagan et al., Phys. Rev. Lett. 85, 5230 (2000).

[7] A. Peres and P. Scudo, Phys. Rev. Lett. 86, 4160 (2001); e-print quant-ph/0010085.

[8] E. Bagan et al., Phys. Rev. A 63, 052309 (2001); e-print quant-ph/0012006.

[9] The relevant group here is $S U(2) / U(1)$, where $U(1)$ $=\left\{e^{i \mathbf{n} \cdot \mathbf{S} \psi} \mid 0 \leqslant \psi<4 \pi, \vec{n}\right.$ fixed $\}$ is the set of "rotations" around $\vec{n}$. By integrating out $\psi$, any encoding state becomes a statistical mixture whose density matrix commutes with $\vec{n} \cdot \vec{S}$. Hence, it is a convex sum of projectors onto the eigenspaces of $\vec{n} \cdot \vec{S}$. No such convex sum can lead to a larger fidelity than its optimal projector, so it is sufficient to consider only eigenstates of $\vec{n} \cdot \vec{S}$.

[10] A. S. Holevo, Probabilistic and Statistical Aspects of Quantum Theory (North Holland, Amsterdam, 1982).

[11] Y. Ayant and M. Borg, Fonctions Speciales (Dunod, Paris, 1971), Sect. 13.6.

[12] A. Peres, Quantum Theory: Concepts and Methods (Kluwer, Dordrecht, 1995), Chap. 9.

[13] A. R. Edmonds, Angular Momentum in Quantum Mechanics (Princeton University, Princeton, NJ, 1960). 